\begin{tabular}{|l|c|c|c|}
\hline Eiszeitalter u. Gegenwart & $\mathbf{4 8}$ & $\begin{array}{c}38-49 \\
\text { Abb., 2 Tab. }\end{array}$ & Hannover 1998 \\
\hline
\end{tabular}

\title{
Zur Geologie der Deckschichten von Kärlich/Mittelrhein
}

\author{
Wolfgang Boenigk \& Manfred Frechen*)
}

\author{
Middle Pleistocene, terraces, loess stratigraphy, interglacial paleosoils, \\ heavy minerals, Rhineland, West Germany
}

Kurzfassung: Das Profil der Tongrube Kärlich wurde geologisch neu bearbeitet, mit folgenden Ergebnissen:

1. Die Einstufung von Abschnitt $\mathrm{A}$ in den JaramilloEvent ist nicht bewiesen. Abschnitt A kann jünger sein und damit in den Bavel-Komplex gehören.

2. Der quartäre Vulkanismus der Osteifel setzt in der Warmzeit ein, die Abschnitt $\mathrm{F}$ abschließt. Es gibt Hinweise im Profil von Kärlich, daß in der Westeifel der quartäre Vulkanismus schon deutlich früher, schon in der Matuyama-Epoche, einsetzte.

3. Eine Unterteilung von Abschnitt G, im Sinne von RAZI RAD (1976), gibt es nicht und hat es nicht gegeben. Alle Sedimente von Abschnitt G zeigen eine Dominanz von brauner Hornblende im Schwermineralspektrum.

4. Abschnitt $\mathrm{H}$ ist durch die markanteste Diskordanz des gesamten Profils in Abschnitt H-unten und Abschnitt H-oben geteilt.

5.Das „Kärlicher Interglazial“ ist zu unterteilen in ein Kärlicher Interglazial I, unter dem Brockentuff und ein Kärlicher Interglazial II, über dem Brockentuff. Die beiden Interglaziale sind durch mindestens eine Kaltphase voneinander getrennt.

\section{[To the geology of the cover sediments of Kärlich/Mittelrhein]}

Abstract: The section at Kärlich is one of the most important localities in Germany where Pleistocene fluvial sand and gravel as well as loess and loess derivatives are exposed. Based on recent geological investigations the stratigraphy of the cover sediments and the local stratigraphic correlations have to be reinterpreted. Our investigations indicate that:

1. The correlation of Unit "Abschnitt" A with the Jaramillo event is under discussion. It ist likely that Unit $\mathrm{A}$ is younger and hence, correlated with the Bavelium complex.

2. The Quaternary volcanism of the East Eifel Volcanic Field began during the interglacial which tops Unit F. However, the volcanism of the West Eifel Volcanic Field began much earlier, most likeley during the Matuyama chron.

3. There are no further subunits in Unit G, as proposed by Razi Rad (1976), indicated by a dominance of brown hornblende in the heavy mineral spectra for all sediments.

4. Unit $\mathrm{H}$ is subdivided into Unit $\mathrm{H}$-lower part and Unit H-upper part by a marked unconformity.

*) Anschrift der Verfasser: Prof. Dr. W. Boenigk \& Dr. M. FRECHEN, Geologisches Institut der Universität zu Köln, Zülpicher Straße 49a, 50674 Köln
5. The "Kärlich Interglacial" has to be subdivided into the Kärlich Interglacial I from below "Brockentuff" (phreatomagmatic tephra deposits) and a Kärlich Interglacial II from above this tephra layer. The two interglacials are separated by at least one cold phase or interstadial.

Die Deckschichten in der Tongrube Kärlich (Abb.1) wurden 1969 von BrunNaCKer et al. umfassend neu beschrieben und sind seitdem als vielgliedriges Standardprofil für das Mittelpleistozän des Rheinlandes international bekannt. In der über 25jährigen Folgezeit wurden immer wieder Einzelbeobachtungen veröffentlicht, von denen vor allem die Bearbeitung der Flora des Kärlicher Interglazials (BITTMANN 1990, 1991), der Fauna aus Abschnitt G (KolfsChOTEN 1990, KolfsChOten et al. 1990, KOlfSChOten \& Turner 1986) und Datierungen der vulkanischen Einschaltung mit physikalischen Methoden (BOGAARD \& SCHMINKE 1988, 1990, BOGAARD et al. 1989) zu nennen sind. BoGAARD et al. (1989) kommen aufgrund ihrer Datierungen der Tephralagen im Deckschichtenprofil von Kärlich zu der Auffassung, daß erhebliche Teile der Abfolge neu interpretiert werden müssen. Infolge des fortgeschrittenen Abbaus seit Ende der 60er Jahre ist die Verknüpfung der neuen Beobachtungen mit den alten Profilbeschreibungen nicht immer fehlerfrei. Es erfolgte daher eine vollständige geologische Bestandsaufnahme für die älteren Ablagerungen unter der Bezeichnung „Kärlich-Hauptwand" (Abb.2) (BOENIGK \& FrECHEN 1998 a) und für die jüngere Abfolge von der Lokalität „Kärlich-Seeufer” (BOENIGK \& FrecheN 1998b). Die Hauptergebnisse dieser Arbeiten werden im folgenden zusammenfassend dargestellt. Parallel zu den geologischen Untersuchungen erfolgt eine paläontologische (KOLFSCHOTEN \& Richter 1998) und eine archäologische Bearbeitung (Vollbrecht 1998, Gaudinski 1998). Die Beschreibung der Schichten erfolgte entsprechend der Unterteilung nach BRUNNACKER et al. (1969), die sich über Jahrzehnte bewährt hat. Die Abschnitte werden als lithologische Einheiten definiert und die Abgrenzung nicht durch klimastratigraphische Grenzen festgelegt. 


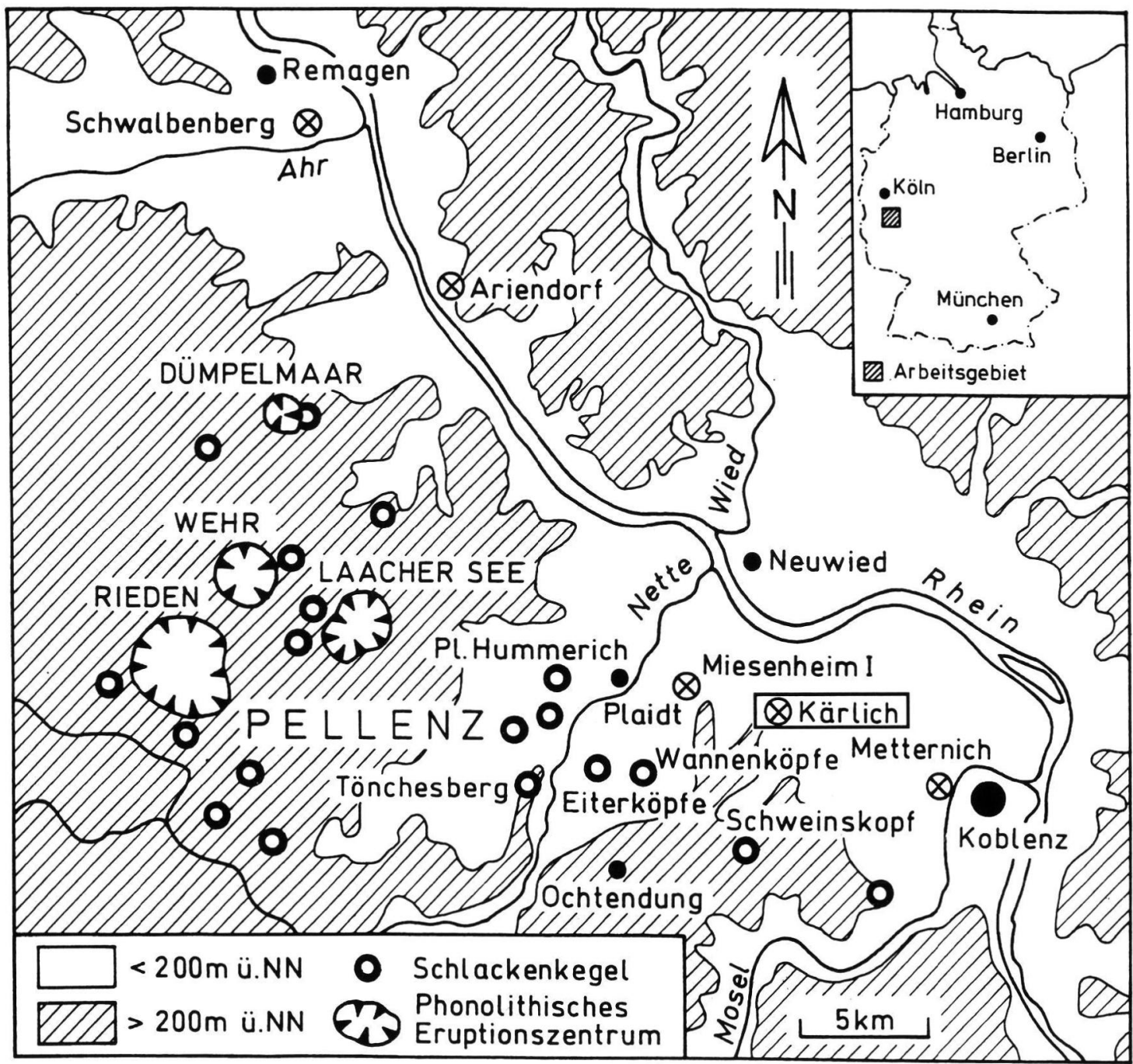

Abb.1: Übersichtsskizze zur Lage des Arbeitsgebietes

Fig. 1: Map showing the area of interest and the location of the section at Kärlich in the Neuwied basin, Germany.

Abb.2: Skizze der Abbauwände der quartärzeitlichen Sedimente in der Tongrube Kärlich mit der Hauptabbauwand und dem Profil des Kärlicher Interglazial I und dem Abbauareal von „Kärlich Seeufer" mit der archäologischen Grabung und dem Profil des Kärlicher Interglazial II

Fig. 2: The section at Kärlich consists of two main areas of interest, the main wall with the profile of Kärlich Interglacial I and the excavation area of "Kärlich Seeufer" with the archaeological find horizon and the profile of Kärlich Interglacial II.

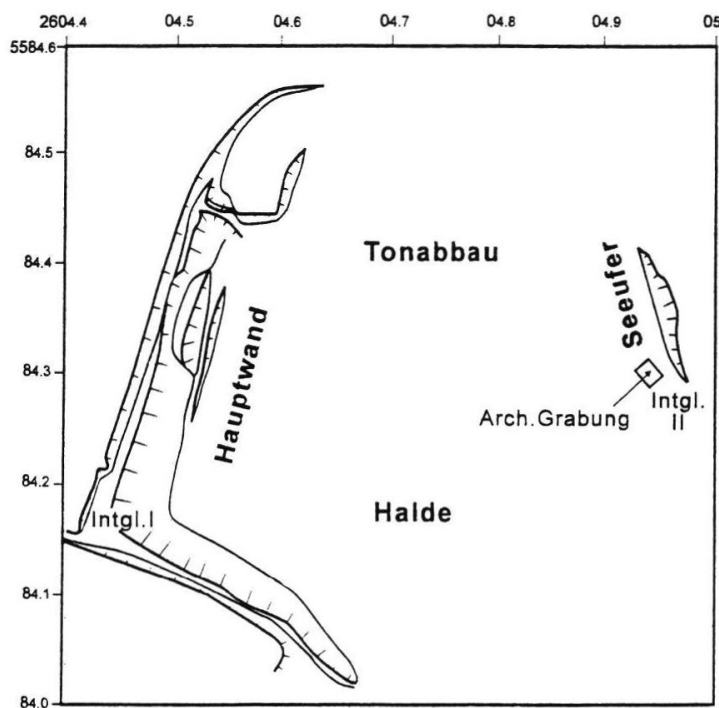




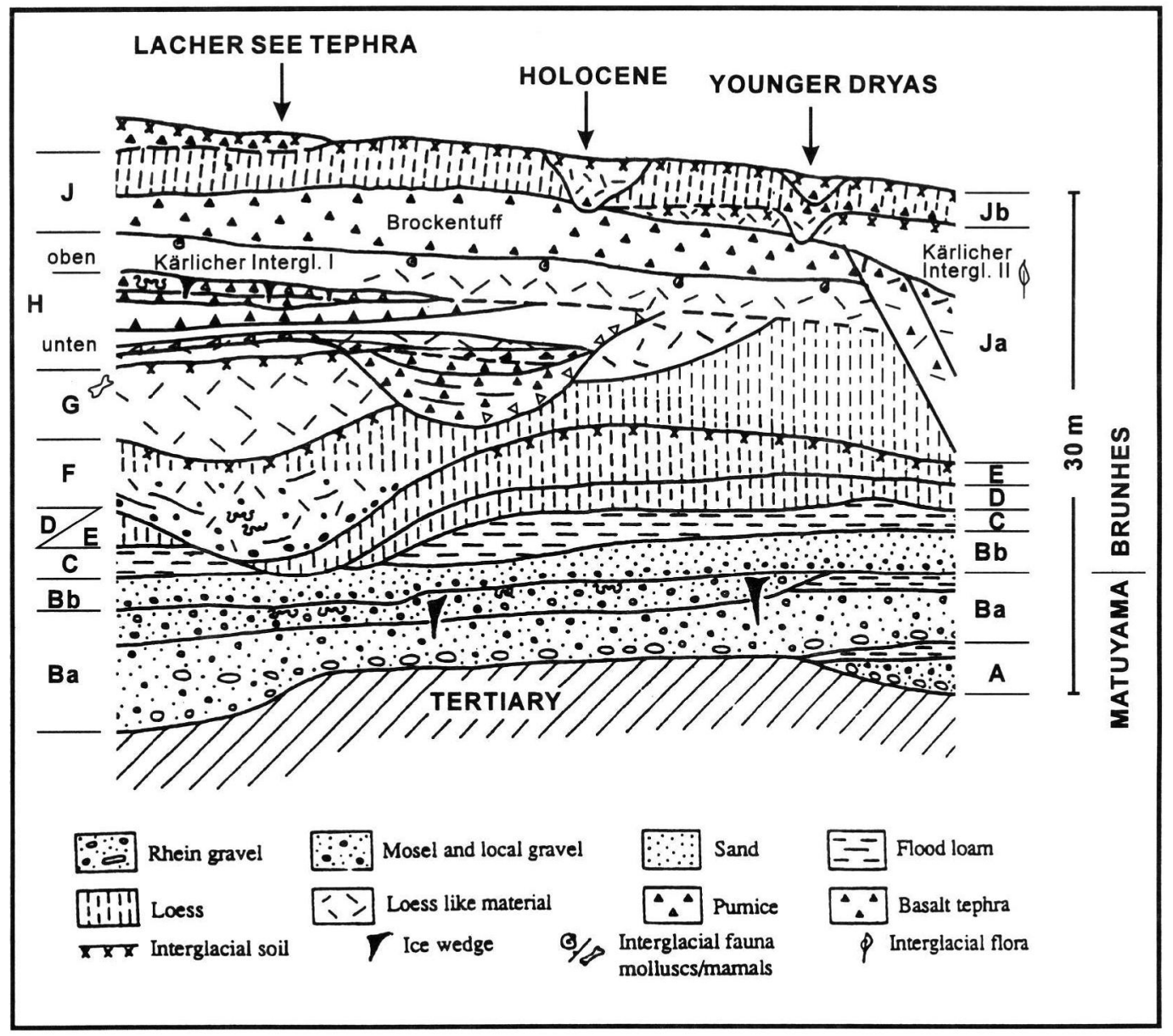

Abb. 3: Schema der quartären Deckschichtenfolge in der Tongrube Kärlich

Fig. 3: Stratigraphic sequence of the cover sediments from the clay pit at Kärlich.

\section{Abschnitt A}

Die Sedimente des Abschnitts A (Abb. 3) werden als Rinnenablagerungen, eingetieft in den tertiären Knubbletten, beschrieben (Mordziol 1930, FRECHEN \& VILLWOCK 1959, BRUNNACKER et al. 1969, Kočı et al. 1973, Boenigk 1978, Fromm 1987, BoeNIGK \& FRECHEN 1998a). BOGAARD et al. (1989) beschreiben Sedimente des Abschnitts A als tektonisch verstellt, und zwar soll dieses tektonische Ereignis zwischen der Ablagerung der Sedimente von Abschnitt A und B stattgefunden haben. BoENIGK \& FRECHEN (1998a) lehnen diese Interpretation ab und führen die Verstellung auf Hangrutsch im Spät- bis Postglazial zurück.

Zur Petrographie liegen aus den Sedimenten des Abschnitts A Geröllanalysen von FrECHEN \& VILLwoCk (1959), BRUNNACKer et al. (1969), RAzi RAD (1976) und BoENIGK (1978) vor. Die Sandfraktion ist bei Frechen \& VilLwOck (1959) beschrieben, und spezielle Analysen zur Schwermineralführung finden sich bei FreCHEN \& VILLwOCK (1959), Razi RAD (1976), Boenigk (1978) und BoeNIGK \& FRECHEN (1998a).

Die Schotteranalysen ergeben einen durchschnittlichen Quarzgehalt in der Fraktion 20-50 $\mathrm{mm} \varnothing$ von $58 \%$ und sind durch Lokalmaterial geprägt.

Die Schwermineralanalysen haben bei allen Bearbeitern hohe Anteile an Epidot erbracht, ein wesentliches Indiz für die Ansprache als unterpleistozäne Rheinablagerungen. Darüber hinaus wird bei BoenIGK \& Frechen (1998) ein Moseleinfluß beschrieben, der mit einer geringen Pyroxenführung verbunden ist. Diese vulkanischen Minerale deuten darauf hin, daß schon zu dieser Zeit in der Westeifel quartärer Vulkanismus existierte und der Osteifelvulkanismus erst wesentlich später einsetzte. 
Zur Altersbestimmung wurden neben der Analyse der petrographischen Zusammensetzung paläomagnetische Messungen durchgeführt (Koči et al. 1973, Boenigk et al. 1974 und Fromm 1987). Es wurde in allen drei Bearbeitungen methodisch unterschiedlich vorgegangen, dennoch ist das Ergebnis gleich: die feinkörnigen Sedimente sind invers magnetisiert. In den gröberen klastischen Ablagerungen im tieferen Teil und den stärker pedogen überprägten Schluffen im höchsten Teil treten normale magnetische Feldrichtungen auf. Diese können entweder als sekundäre Magnetisierung oder als echte Feldumkehr interpretiert werden.

An Fossilien wurde ein Zahn von Hippopotamus $s p$. aus dem Abschnitt A genannt (BRUNNACKer et al. 1980). Die sedimentologischen Befunde sprechen für ein mäandrierendes Gewässer. Sedimentologie und Fauna zusammen deuten auf interglaziale Verhältnisse zur Zeit der Ablagerung. Die normalen magnetischen Feldrichtungen im höheren Teil können als Jaramillo-Event interpretiert werden. Bei dieser Deutung muß jedoch mit einer großen zeitlichen Lücke zum hangenden Abschnitt B gerechnet werden, die im Zusammenhang mit der Grobblocklage an der Basis des Haupt- bzw. Rhein-Schotters gesucht werden kann. Es bleibt jedoch auch die Möglichkeit, daß die gemessenen normalen magnetischen Feldrichtungen auf sekundäre Effekte zurückzuführen sind und dieses Interglazial des Abschnitts $\mathrm{A}$ in den Zeitraum zwischen Jaramillo-Event und Matuyama/Brunhes-Grenze einzustufen ist.

\section{Abschnitt B}

Der ca. $8 \mathrm{~m}$ mächtige, im gesamten Grubenbereich durchhaltende Schotterkörper (Abb. 3) ist komplex aufgebaut, wie schon die Beschreibung bei Brunnacker et al. (1969) und Boenigk et al. (1974) zeigen (Tab.1).

Wie Tab.1 zeigt, besteht Abschnitt B aus 4 eigenständigen Schotterpaketen, die durch Hochflutlehm mit Auenboden bzw. Gley oder Diskordanz mit Blocklage voneinander getrennt werden.

Schotteranalysen aus Abschnitt B finden sich bei Brunnacker et al. (1969) und Boenigk (1978). Die Schwermineralfraktion wurde von FRECHEN \& v.d. Boom (1959), Frechen \& Heide (1969), Razi Rad (1976) und Boenigk (1978) untersucht. Die petrographischen Analysen ergeben, daß man einen älteren Rheinschotter von einem jüngeren Moselschotter und einem dazwischen liegenden Mischschotter unterscheiden kann. Beim Moselschotter ist eine deutliche vulkanische Komponente im
Schwermineralspektrum erkennbar, die, wie für Abschnitt A, auf quartären Vulkanismus in der Westeifel zurückgeführt wird.

Tab. 1: Gliederung des quartären Terrassenschotters in Kärlich nach BrunNACKER et al. (1969) und BoENigK et al. (1974)

Table 1: Schematic sketch of the Quaternary terrace sediments after BrunNacker at al. (1969) and BoeNIGK et al. (1974).

\begin{tabular}{l|l}
\hline BRUNNACKER et al.(1969) & BoENIGK et. al. (1974) \\
\hline $\begin{array}{l}\text { Abschnitt C } \\
\text { Hochflutlehm }\end{array}$ & $\begin{array}{l}\text { Gley } \\
\text { Hochflutlehm }\end{array}$ \\
\hline $\begin{array}{l}\text { Auenboden } \\
\text { Hochflutlehm } \\
\text { Oberer Schotter }\end{array}$ & $\begin{array}{l}\text { Auenboden } \\
\text { Hochflutlehm } \\
\text { Moselschotter } \\
\text { (oberer Schotter) }\end{array}$ \\
\hline & $\begin{array}{l}\text { brauner Auenboden } \\
\text { lehmiger Hochflutsand } \\
\text { Rhein-Mosel-Misch- } \\
\text { schotter mit Eiskeil- } \\
\text { pseudomorphosen } \\
\text { und Kryoturbationen }\end{array}$ \\
\hline $\begin{array}{l}\text { Abschnitt B } \\
\text { Gley }\end{array}$ & $\begin{array}{l}\text { Gley } \\
\text { Hochflutlehm } \\
\text { Hauptschotter } \\
\text { Blocklage }\end{array}$ \\
\hline Unterer Schotter & Bheinschotter \\
\hline $\begin{array}{l}\text { Abschnitt A } \\
\text { Auenboden, stark } \\
\text { pseudovergleyt }\end{array}$ & nicht erschlossen \\
Schotter & $\begin{array}{l}\text { Auenboden, stark } \\
\text { pseudovergleyt } \\
\text { Schotter }\end{array}$ \\
\hline
\end{tabular}

Zur Altersbestimmung wurden die feinkörnigen Lagen des Abschnitts B mit verschiedenen Methoden paläomagnetisch untersucht (HEYE in BOENIGK et al. 1974, KoČI in BRUnNaCKer et al. 1976, Fromm 1987). Übereinstimmend wird über eine inverse Magnetisierung der Rheinschotter berichtet. KočI (zit. in Boenigk 1978) gibt inverse Magnetisierung für die Hochflutsande auf dem Rhein/Mosel-Mischschotter an. Der die Terrasse abdeckende Hochflutlehm und die überlagernden Deckschichten werden übereinstimmend in allen drei Bearbeitungen als eindeutig normal magnetisiert beschrieben. Bei den Ergebnissen der Messungen an dünnen Sandlinsen in den Schottern muß mit einer nachträglichen Umorientierung der magnetischen Feldrichtung gerechnet werden. Damit bietet sich an, die Umkehr des 
Magnetfeldes von der Matuyama- zur BrunhesEpoche nach der Sedimentation des Hochflutsandes auf dem Mischschotter und vor der Ablagerung des Hochflutlehms auf dem Moselschotter zu suchen.

Eindeutig glaziale Klimaverhältnisse sind nur für den Mischschotter durch Eiskeilpseudomorphosen und Kryoturbationen belegt. Die zahlreichen Driftblöcke an der Basis von Abschnitt B sind ebenfalls Hinweise auf kaltes Klima. Die Hochflutlehme mit ihrer pedogenen Überprägung belegen dagegen Abschnitte günstigerer Klimabedingungen.

\section{Abschnitt C - D - E}

Über diesen unteren Teil der Deckschichten (Abb. 3) gibt es wenig neue Information. Es handelt sich im wesentlichen um verspülte lößartige Sedimente mit Solifluktionsdecken (SGIBNEV 1998, BoENIGK \& FreCHEN 1998a) mit zwischengeschalteten relativ schwachen Bodenbildungen. Äolische Ablagerungen sind nicht vorhanden. Die Zusammensetzung des Schwermineralspektrums (RAZI RAD 1976, BOENIGK \& FreCHEN 1998a) wird stark durch die Körnung bestimmt. Feinkörnige Sedimente führen sehr viel Zirkon, sandige zeigen dagegen eine Dominanz des Rheinspektrums mit vorherrschend Epidot, gefolgt von Granat, grüner Hornblende und Alterit. Die Fauna, sowohl die Kleinsäugerfauna wie die Molluskenfauna (KOLFSCHOTEN \& RiCHTER 1998), enthalten interglaziale und glaziale Elemente und weisen, wie die Sedimente, auf Umlagerung und Verspülung hin.

Der Profilabschnitt C - E ist durch wiederholte Erosion-Akkumulation und Bodenbildung gekennzeichnet. Eindeutige interglaziale und hochglaziale Ablagerungen sind nicht vorhanden.

\section{Abschnitt F}

Mit der Ablagerung des Abschnitts F tritt im Profil von Kärlich zum ersten Mal Löß auf. Es wurde Schwemmlöß und echter äolischer Löß akkumuliert. Die Ablagerungen sind relativ wenig gegliedert. Im tieferen Teil kommen Sand- und Kieslagen vor. Die Kiese an der Basis bestehen aus aufgearbeitetem Kieseloolith-Material. Die Sande dagegen sind von pleistozäner rheinischer Fazies, z.T. fluviatil, im höheren Bereich aber auch äolisch, abgelagert. Neben mehreren schwachen Naßböden unterteilt ein markanter brauner
Boden Abschnitt F. Die Schwermineralanalysen (RAZI RAD 1976, Boenigk \& Frechen 1998a) ergaben, daß Abschnitt F durch die Minerale Zirkon, Epidot, Granat und grüne Hornblende charakterisiert ist. Vulkanische Schwerminerale fehlen. Diese treten zum ersten Mal im Bt der Abschnitt F abschließenden Bodenbildung auf.

Bei Brunnacker et al. (1969) und Bogatrd et al. (1989) wird von einem Tuffband in Abschnitt $F$ berichtet. Das wäre der Hinweis auf die erste vulkanische Tätigkeit während des Quartärs in der Osteifel. Die Bearbeitung der Lößprofile am Mittelrhein hat jedoch ergeben, daß vulkanische Schwerminerale nach dem Einsetzen der vulkanischen Aktivität in der Osteifel durch Verwehung, Verspülung und solifluidale Verlagerung in den Deckschichten allgemein und mit großer Häufigkeit verbreitet sind. Das ist in Abschnitt F nicht der Fall. Daraus ergibt sich, daß das Vorhandensein eines Tuffbandes in Abschnitt $\mathrm{F}$ angezweifelt werden muß. Für den Tuff KAE-BT1 (BogaARd et al. 1989, Schirmer 1990) konnte nachgewiesen werden, daß dieser Tuff nicht in Abschnitt F, sondern in Abschnitt $G$ liegt (BOENIGK \& FrecheN 1998a). Für die Tuff-Linse in Abschnitt $F$ bei BRUNNACKER et al. (1969) ist eine Überprüfung nicht mehr möglich. Man könnte dabei an eine Apophyse des Brockentuffs denken. Solche Apophysen sind in der Grube seit Mitte der 80er Jahre fast durchgängig an der Grenzfläche Tertiär/Quartär beobachtet worden. Sie sind bei BogAARD et al. (1989) beschrieben. Solche Apophysen wurden auch in jüngeren Schichten nachgewiesen, auch in Abschnitt F.

Abschnitt $\mathrm{F}$ ist mit seiner ausgeprägten Lößakkumulation eindeutig einem Glazial zuzuordnen. Mehrere Naßböden und ein brauner Boden belegen Klimaschwankungen. Die Fauna (BrunNACKER 1971, KOLFSCHOTEN \& Richter 1998) deutet auf glaziale Steppenbedingungen. Bei BRunNACKER et al. (1969) wird unter Kaltklimaindikatoren das Auftreten von Eiskeilen erwähnt. Die Formen werden aber nicht beschrieben, weder im Text noch in den Abbildungen. Diese Eiskeile werden in späteren Arbeiten (SCHIRMER 1990, 1995) zitiert. Da die Sedimente des Abschnitts F unbestritten glazialzeitlich sedimentiert wurden, ist die Möglichkeit, daß Permafroststrukturen auftreten, durchaus gegeben. Als Beweis sollten die erwähnten Eiskeilpseudomorphosen jedoch so lange nicht verwendet werden, bis eine genaue Beschreibung und Lokalisierung vorliegen. Die in den letzten Jahren zu beobachtenden Spalten 
sind auf Setzungserscheinungen zurückzuführen (BoenigK \& Frechen 1998a). Sie sind damit keine Klimaindikatoren.

\section{Abschnitt G}

Bei den Ablagerungen des Abschnitts $G$ handelt es sich um eine bis $7 \mathrm{~m}$ mächtige Rinnenfüllung (Abb. 3) von dunkelbraunen Lehmen, die in 5 Rhythmen gegliedert ist (BOENIGK \& FrECHEN 1998a). Mehrere dieser Rhythmen sind durch kräftige Bt-Horizonte überprägt.

Petrographisch ist Abschnitt $G$ durch die Dominanz der vulkanischen Minerale im Schwermineralspektrum gekennzeichnet, und zwar mit einer Vorherrschaft der braunen Hornblende über die Pyroxene, die ihrerseits in den jüngeren Ablagerungen dominieren In Abschnitt $G$ tritt der erste pleistozäne Tuffhorizont der gesamten Abfolge auf. Es ist der Horizont, der bei BOGAard et al. (1989) als KAE-BT1 beschrieben ist (vgl. BoEnIGK \& FRECHEN 1998a).

Schwermineraluntersuchungen von RAZI RAD (1976) führten zu einer Untergliederung des Abschnitts $G$ in Abschnitt Ga: Abschnitt ohne vulkanische Schwerminerale und Abschnitt Gb: Sedimente mit Dominanz von brauner Hornblende im Schwermineralspektrum.

Boenigk \& Frechen (1998a) konnten zeigen, daß seit der interglazialen Bodenbildung auf Abschnitt $\mathrm{F}$ die braune Hornblende durchgängig in Abschnitt $G$ dominiert. Ein Abschnitt $G a$ ist im Gelände von keinem Bearbeiter beschrieben worden. Die Analysen von RAzI RAD (1976) wurden an Archivmaterial durchgeführt. Eine Verknüpfung dieser Proben mit in der Literatur beschriebenen Profilen ist nicht möglich. Es muß angenommen werden, daß es zu einer Verwechslung der Zuordnung der Proben gekommen ist. Die Untergliederung von Abschnitt $G$ aufgrund der Schwermineralführung hat keinen realen Hintergrund und muß ersatzlos zurückgenommen werden. Die bei Kolfschoten et al. (1990) und KOLFSCHOTEN \& TURNER (1996) vorgenommene Unterteilung von Abschnitt $G$ erfolgte anhand des Tuffhorizontes KAE-BT1 in einen darunter liegenden Teil und einen jüngeren Teil. Diese Aufteilung hat nichts mit der Gliederung bei RAZI RAD (1976) zu tun, auch wenn die Bezeichnungen Ga und $\mathrm{Gb}$ verwendet werden.

Für eine klimatische Interpretation der durch Abschnitt $G$ repräsentierten Zeitspanne stehen als Befunde Fließerden und Abspülsedimente als Indikatoren einer offenen Vegetation einerseits und Parabraunerden als Waldböden und warmzeitliche Klein- und Großsäugerfunde (BRunNACKER 1971, KOLfSCHOTEN et al. 1990, TurNER 1989, KOLFSCHOTEN \& Richter 1998) andererseits zur Verfügung. Es finden sich aber auch Faunenelemente, die auf Steppenvegetation deuten (KolfSCHOTEN \& RiCHTER 1998). Die Molluskenfauna (LOŽEK in BRUNNACKER 1971) deutet ebenfalls auf eine Lößsteppe als Lebensraum. Das Vorhandensein von Eiskeilen in Abschnitt G (BRUNNACKer et al. 1969, SCHIRMER 1990, 1995) ist nicht gesichert. Es liegt keine Beschreibung oder Abbildung vor. Bei Berücksichtigung aller Informationen zum Klima kann man feststellen, daß Beweise hochglazialer Bedingungen nicht vorhanden sind, dagegen finden sich viele Hinweise auf interglaziale bis interstadiale Umweltverhältnisse. Man könnte die gesamte Abfolge von Abschnitt $G$ als Sediment einer Warmzeit interpretieren, wobei die Umlagerungen der Sedimente und Beimischung von kaltklimatischen Faunenelementen durch eine durch lokalen Vulkanismus verursachte instabile Landoberfläche zu erklären wäre.

Der Interpretation als Ablagerung nur einer Warmzeit stehen zwei Überlegungen entgegen:

1. Die Bodenbildungen auf Abschnitt $F$ und in Abschnitt G sind so kräftig, daß zu ihrer Genese eine längere Zeit benötigt wird.

2. Der Übergang von Mimomys in Abschnitt F zu Arvicola in Abschnitt G (KOLFSCHOTEN \& RichTER 1998) sollte ebenfalls einen längeren Zeitabschnitt repräsentieren.

\section{Abschnitt H}

Im Gegensatz zum kräftig gefärbten Abschnitt G ist Abschnitt H stärker durch graue Farbtöne gekennzeichnet. Abschnitt $\mathrm{H}$ ist petrographisch im Gegensatz zu Abschnitt G durch das Einsetzen der Vorherrschaft der Klinopyroxene im Schwermineralspektrum charakterisiert. Die Obergrenze von Abschnitt $\mathrm{H}$ wird wie bei BRUNNACKER et al. (1969) an die Basis des Kärlicher Brockentuffs gelegt (Abb. 3), der im gesamten Aufschlußbereich einen gut faßbaren Leithorizont darstellt. Die Erweiterung des Abschnitts $\mathrm{H}$ auch auf Sedimente im Hangenden des Brockentuffs (Bosinski et al. 1980, SCHIRMER 1990, 1995) wird abgelehnt, weil dadurch eine klare Abgrenzung unmöglich wird. Die Ablagerungen von Abschnitt $\mathrm{H}$ bestehen aus mehreren Rhythmen von Erosion, Verspülung, Fließerde, z. T. Löß und Bodenbildung und werden durch eine ausgeprägte Diskordanz (Abb. 3) in einen unteren und einen oberen Abschnitt un- 
terteilt. Die auffälligste Erscheinung in Abschnitt $\mathrm{H}$ sind die vulkanischen Tuffe aus Bims- und Basalt-Material.

Für die phonolithischen Tephren aus dem Abschnitt $\mathrm{H}$ liegen ${ }^{40} \mathrm{Ar} / 39 \mathrm{Ar}-D a t i e r u n g e n ~ v o r$ (LipPOlT et al. 1986, BOGAARD \& SCHMINCKE 1988, 1990, BogaArd et al. 1989, Schmincke 1994), die die Ablagerungen des Abschnitts $\mathrm{H}$ in den Zeitraum um 450 ka datieren. Glaziales Klima ist für Abschnitt $\mathrm{H}$ in mehreren Horizonten durch Löß, Froststrukturen und Eiskeile nachgewiesen. Die Bodenhorizonte deuten auf wiederholte warmzeitliche Bedingungen (BOENIGK \& FRECHEN 1998a). Interglaziale Bedingungen werden nur im höheren Teil im „Kärlicher Interglazial“ erreicht.

Frechen \& Rosauer (1959) und MüCKEnhausen (1959) beschreiben unter dem Brockentuff eine Braunerde und BrunNaCKer et al. (1969) eine Parabraunerde. Diese Bodenbildung ist in den letzten Jahren im Aufschluß Kärlich nur noch mit dem Kalkanreicherungshorizont und der dazugehörigen Molluskenfauna in der hangenden Fließerde erhalten. Der A- und auch der B-Horizont sind während der starken Abtragungsphase, die H-unten von H-oben trennt (Abb.3), entfernt worden. Die Molluskenfauna wird von LožEK (in BRUNNACKER 1971) und ROTH (1995) als warmzeitlich beschrieben.

Die Sedimente von Abschnitt $\mathrm{H}$-oben verlangen eine besondere Beachtung, da diese Ablagerungen die Typ-Sedimente des „Kärlicher Interglazials" (BRUNNACKER et al. 1975) nach BRUNNACKER et al. (1969) und BRUNNACKER (1971) darstellen.

Von Urban (1978) und Bosinski et al. (1980) wurde der Begriff „Kärlicher Interglazial“ auch auf Sedimente im Hangenden des Kärlicher Brockentuffs übertragen. Da diese Sedimente pollenanalytisch untersucht werden konnten, wurde der Begriff „Kärlicher Interglazial“ zunehmend auf diese jüngeren Ablagerungen bezogen. Um Mißverständnissen vorzubeugen, wurde von BOENIGK (1995a, b) vorgeschlagen, das ursprünglich identifizierte Interglazial im Liegenden des Brockentuffs (BrunnaCKer et al. 1969, BrunNaCKer 1971), belegt durch eine Parabraunerde und eine interglaziale Molluskenfauna in der darüber liegenden Fließerde aus Bodenmaterial als „Kärlicher Interglazial I" und das Interglazial, das pollenanalytisch in den Sedimenten im Hangenden des Kärlicher Brockentuffs nachgewiesen ist, als „Kärlicher Interglazial II“ zu bezeichnen. Zwischen diesen beiden warmklimatischen Phasen liegen nach dem heutigen Kenntnisstand mindestens (BOENIGK \& FRECHEN 1998a):
- eine Erosionsphase, die die Parabraunerde bis auf den Cc-Horizont kappt

- die Sedimentation einer Fließerde aus Bodenmaterial mit einer Molluskenfauna eines trockenen, warmen Klimas

- eine schwache pedogene Überprägung mit intensiver Bioturbation

- die Sedimentation einer weiteren Fließerdedecke mit einer Molluskenfauna eines interglazialen Waldes

- eine geringe Pseudovergleyung der Fließerde

- die Ablagerung des Kärlicher Brockentuffs

- der Versturz des Kärlicher Brockentuffs mit Bildung der Depression, in der die Sedimente des „Kärlicher Interglazials II“ liegen.

Aus dem Brockentuff wurden 4 Phlogopit-Einzelkristalle aus dem Kärlicher Brockentuff (KAE-

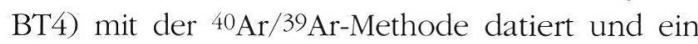
Alter von $396 \pm 20 \mathrm{ka}$ ermittelt (BOGAARD et al. 1989). Die Eruption würde damit in der zweiten Hälfte des Interglazials von „stage 11“ der Sauerstoff-Isotopenkurve liegen, und man müßte eine voll entwickelte Parabraunerde im Liegenden vorfinden. Das ist aber nicht der Fall. Mikromorphologische Bodenuntersuchungen geben keinerlei Hinweis auf eine Braunerde oder $\mathrm{Pa}$ rabraunerde (Boenigk et al. 1998). Der Geländebefund zeigt, daß schwach pedogen überprägte Fließerden mit umgelagerten warmzeitlichen Mollusken zu finden sind, die die Ablagerungen einem Frühglazial zuordnen. Dieser Befund wird bestätigt durch eine neuere Datierung durch BoGAARD (Vortrag: DFG-Abschlußkolloquium zum "Quartär der Eifel“ am 11. 6. 1997), die den Brockentuff in den Anfang von "stage 10" (Abb. 4) stellt. Damit müssen „Kärlicher Interglazial I“ und „Kärlicher Interglazial II“ streng voneinander getrennt werden. Die warmklimatischen Florenreste, von denen Schirmer (1990) von der Basis des Brockentuff berichtet, können als kurze Klimabesserung in einem Interstadial angesprochen werden.

\section{Abschnitt J}

Abschnitt $\mathrm{J}$ beginnt mit dem im gesamten Grubenbezirk vertretenen mehrere m-mächtigen Kärlicher Brockentuff. Darüber folgt in der Regel jungweichselzeitlicher Löß mit Resten von Laacher-See-Tuff. Nur im Osten ist die Abfolge komplexer ausgebildet. Als erster beschreibt SCHIRMER (1970) und dann Rohdenburg \& Semmel (1971) eine Deckschichtenfolge, die durch eine $\mathrm{Pa}$ rabraunerde und verschiedene interstadiale Böden untergliedert ist. Seit 1980 waren in der 


\section{SPECMAP Stack}

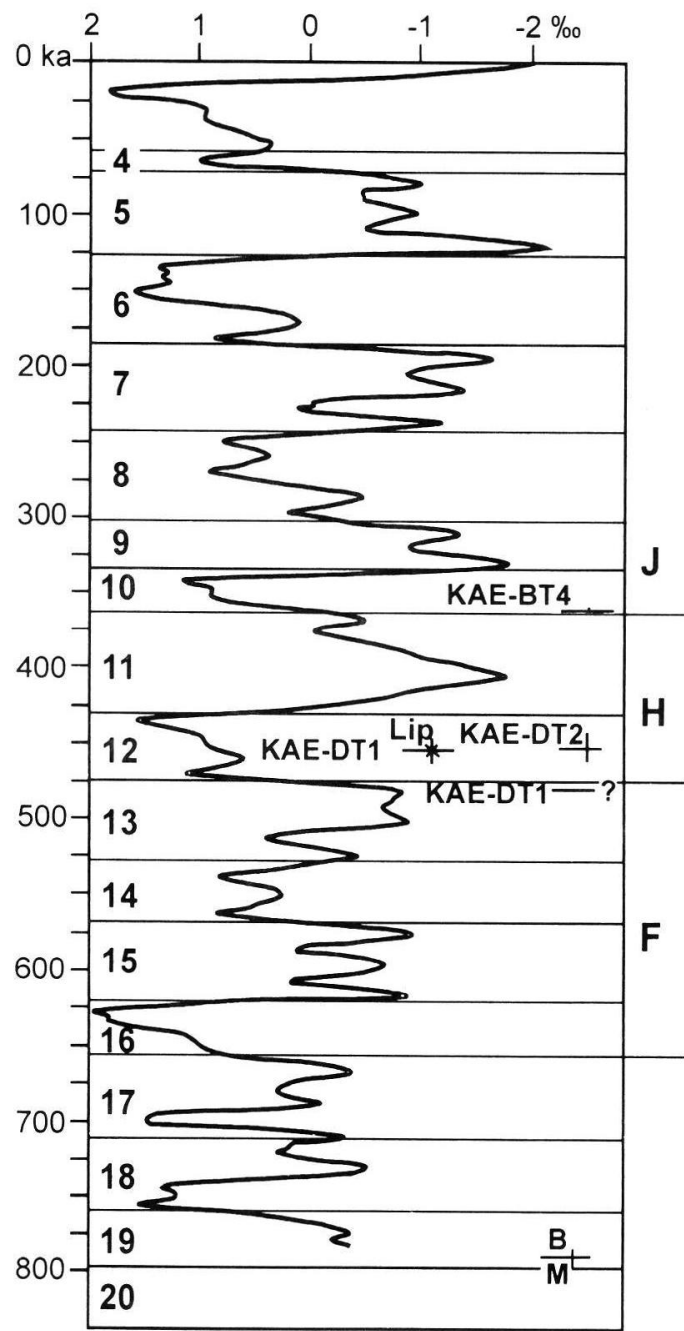

Abb. 4: Die SPECMAP-Sauerstoffisotopenkurve nach Bassinot et al. (1994) mit den Daten der $\mathrm{Ar} / \mathrm{Ar}$-Datierungen aus Kärlich nach Lippolt et al. (1986) und BOGAARD \& SCHMINCKe $(1988,1990)$ sowie v.d. BOGAARD (1997, Vortrag zum DFG-Abschluß-Kolloquium „Quartär der Eifel“). Die Position der M/B-Grenze ist nach TAuxe et al. (1996) eingetragen.

Fig. 4: Chronological results of the Kärlich site; SPECMAP curve after BASSINOT et al. (1994) and B/M boundary after TAuxe et al. (1996), Ar/Ar data from Lippolt et al. (1986), BOGAARD \& SChMincke (1988, 1990) and v. d. BOGAARD (oral presentation during the 1997 seminar "Quaternary of the Eifel Area", German Science Foundation).

Südostecke der Grube in einer Depression humose Sedimente erschlossen, die aufgrund ihres Floren-Inhaltes in eine Warmzeit eingestuft wurden
(Urban 1983, Bittmann 1990, 1991, Kröger et al. 1991, BitTMann \& Müller 1996). Wie oben ausgeführt, müssen diese interglazialen Ablagerungen als „Kärlicher Interglazial II“ bezeichnet werden. Die vollständigste Entwicklung der Sedimentabfolge über dem Brockentuff ist in der Depression in der SE-Ecke der Tongrube erschlossen und als Lokalität „Kärlich Seeufer“ (Abb. 2) bezeichnet. Es wird angenommen, daß die Depression auf vulkanotektonische Ereignisse im Zusammenhang mit phreatomagmatischer Eruption zurückzuführen ist (BOENIGK \& FRECHEN 1998b).

Unmittelbar nach der Bildung der Depression kam es zu Rutschungen, durch die Terrassenschotter, Löß und Brockentuff als Schollen oder als Hangschutt in die Senke verlagert wurden. Danach bildete sich ein See, der mit zum Hangenden feiner werdenden Sedimenten, von Sand über Ton zur rein organischen Mudde gefüllt wurde. Die Mudde ist im zentralen Teil der Senke feinkörnig, am Rand grob mit vielen Holzresten. Auf diese Mudde legen sich Muren oder sandige debris flows in mehreren Paketen. Sie fallen steil von Südwesten in die Senke ein (BOENIGK \& FreCHEN 1998 b), was auf einen Transport von Süden bzw. Südwesten in die Depression deutet. Die einzelnen debris flows sind durch Hiaten voneinander getrennt, was sich durch geringe Aufarbeitung durch fließendes Wasser an der jeweiligen Oberfläche zeigt. Sie haben eine relativ gleichmäßige Mächtigkeit von ca. $1 \mathrm{~m}$, enden mit steiler Stirn und sind übereinander und z. T. nebeneinander gelagert. Das Material der debris flows besteht aus aufgearbeitetem Brockentuff. Es ist schlecht sortiert und zeigt keine interne Schichtung. Die Gesamtmächtigkeit dieser Ablagerungen beträgt maximal $240 \mathrm{~cm}$. Dies ist die archäologische Fundschicht von „Kärlich Seeufer" (GAUDINSKI et al. 1996). Der Abgang der debris flows fällt in eine Phase von Klimaverschlechterung und Übergang der Vegetation vom interglazialen Wald , „Kärlicher Interglazial II“, in eine Waldtundra bis Tundra, wie die Untersuchungen von BitTMann (1991) zeigen.

Über den Ablagerungen der debris flows folgen dunkelgraue Tone und Silte mit mud flows und drei geringmächtigen humusreichen Mudden bzw. Torfen, die in einem Sumpfgebiet mit zeitweise offener Wasserfläche sedimentiert wurden. Klimatisch umfaßt diese Abfolge einen Wechsel von stadialen und interstadialen Bedingungen (BITTMANN 1991). Über der jüngsten Schicht mit hohem organischen Anteil erfolgt ein signifikanter Wechsel im Sediment. Es dominieren braune Farben. Die Ablagerungen bestehen aus gut ge- 


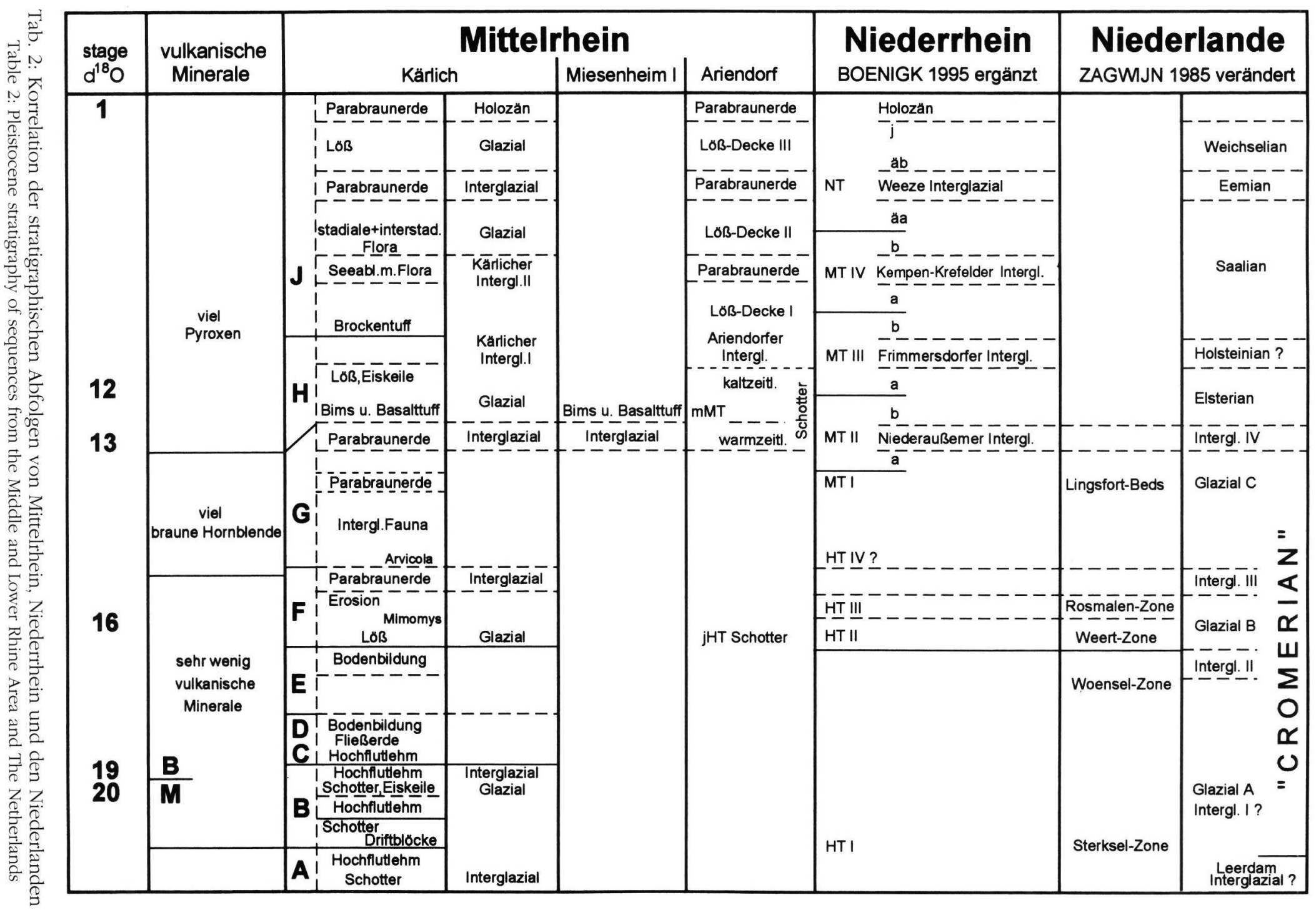


bankten lehmigen Hangabspülungen. Zwei gut erkennbare Pseudogleye sind eingeschaltet. Den Abschluß bildet der Rest eines sehr kräftigen BtHorizontes mit unterlagerndem Kalkanreicherungshorizont. Darüber akkumulierte gelbbeiger, frischer kalkhaltiger Löß bis zur künstlichen Oberfläche.

\section{Chronostratigraphie}

In Tabelle 2 sind die lokalen Stratigraphien vom Mittelrhein den Abfolgen vom Niederrhein und den Niederlanden gegenübergestellt. Die Korrelation erfolgt im ersten Ansatz aufgrund der petrographischen Zusammensetzung der Ablagerungen. Dabei wird vor allem das Auftreten der Schwerminerale des quartären Eifelvulkanismus in den Sedimenten herangezogen.. Der Wechsel im Schwermineralspektrum im Top von Abschnitt F, mit dem ersten Einsetzen des quartären Eifelvulkanismus, und im Top von Abschnitt $G$, mit dem Wechsel der Dominanz von brauner Hornblende zur Dominanz von Pyroxen, liefert zwei Leitmarken. Darüber hinaus wird die Brunhes/Matuyama-Grenze in Abschnitt B zur Datierung der Abfolge herangezogen. Die Verknüpfung des Abschnitts A mit dem Jaramillo-Event scheint nach den vorliegenden Daten möglich, ist aber nicht bewiesen. Ein jüngeres Alter für $\mathrm{Ab}-$ schnitt A, Leerdam Interglazial, ist aus der Sedimentabfolge wahrscheinlicher. Als drittes Argument werden die physikalischen Datierungen der Bimse in Abschnitt $\mathrm{H}$ (BOGAARD \& SCHMINCKe 1988, 1990) herangezogen, die den kaltklimatischen Abschnitt auf ca. $450 \mathrm{ka}$ festlegen und damit eine Korrelation mit stage 12 der Sauerstoffisotopenkurve aus dem marinen Bereich erlauben.

Im nächsten Schritt werden die Klimaindikatoren für die Abfolge von Glazial/Interglazial-Zyklen ausgewertet. Das führt zur Verknüpfung von Abschnitt F, mit seinen eindeutig glazial geprägten Sedimenten, mit stage 16 (Abb. 4). Auf eine weitergehende Verknüpfung der Abfolge von Kärlich mit der Sauerstoffisotopen-Kurve wird verzichtet, da keine eindeutigen Belege vorliegen.

Größere Unstimmigkeiten ergeben sich vor allem bei der Korrelation von Abschnitt J mit den anderen Stratigraphien. Zum einen ist das Alter der Seeablagerungen des Kärlicher Interglazials II unsicher, denn die palynologische Interpretation stimmt nicht mit den Befunden zur Säugerfauna aus Kärlich Abschnitt G. Zum anderen liegen unterschiedliche Ergebnisse zur Datierung des Brockentuffs, der das Kärlicher Interglazial I vom
Kärlicher Interglazial II trennt, vor. Eine neuere physikalische Datierung führt zu einer Einstufung des Tuffs in stage 10 der Sauerstoffisotopenkurve, eine Kaltphase. Die warmklimatischen Ablagerungen von Kärlicher Interglazial I und II sind damit durch mindestens eine Kaltphase voneinander getrennt. Die Schichtenfolge über dem Kärlicher Interglazial II liefert keine genaueren Einstufungsmöglichkeiten. Es scheint festzustehen, wie die Beobachtungen im Aufschluß Kärlich Seeufer und die Arbeiten von Schirmer (1970) und RoHDEMBURG \& SEMmel (1971) beschreiben, daß mindestens ein Interglazial, belegt durch einen kräftigen Bt-Horizont, im Hangenden vom Kärlicher Interglazial II vorhanden ist.

Die Korrelation mit den anderen Stratigraphien (Tab. 2) scheint in sich stimmig. Es ergibt sich aber für das Profil Ariendorf ein Widerspruch zwischen der Deckschichtenstratigraphie und der physikalischen Datierung des Bimses über der jüngsten fossilen Parabraunerde, die ein Alter von 215 ka (BOGAARD \& SCHMINCKe 1990) erbrachte. Dieser Wert datiert die Parabraunerde in ein Interglazial, das älter als Eem-zeitlich ist, was anhand der Deckschichtenfolge nicht zu erwarten ist und auch durch die Säugerfauna dieses Profils nicht gestützt wird (SteEnsma \& Kolfschoten 1998). Man müßte annehmen, daß in allen aufgeführten Stratigraphien im jüngeren Mittelpleistozän ein voller Glazial/Interglazial-Zyklus fehlt. Unterstützt wird diese These durch ein Abzählen der Sauerstoffisotopenstadien. Akzeptiert man das Fehlen eines Klimazyklus, dann ist die Korrelation in Tabelle 2 für das jüngste Mittelpleistozän und Jungpleistozän nicht mehr vertrauenswürdig.

\section{Dank:}

Wir bedanken uns bei der DFG (Bo 413/5-1,2,3) für finanzielle Unterstützung der Forschungsarbeiten.

\section{Literaturverzeichnis}

Bassinot, F. C., Labeyrie L. D., Vincent, E., Quidelleur, X., Shackleton N. J. \& Lancelot, Y. (1994): The astronomical theory of climate and the age of the Brunhes-Matuyama magnetic reversal. - Earth and Planetary Science Letters, 1226: 91-108; Amsterdam.

BrtTMann, F. (1990): Neue biostratigraphische Korrelierung des Kärlicher Interglazials (Neuwieder Becken/Mittelrhein). - In: W. Schirmer (Hrsg.): Rheingeschichte zwischen Mosel und Maas. - Deuqua Führer 1; 67-70; Dormagen. 
- (1991): Vegetationsgeschichtliche Untersuchungen an mittel- und jungpleistozänen Ablagerungen des Neuwieder Beckens (Mittelrhein). - Jahrb. d. Römisch-Germanischen Zentralmuseums Mainz, 38. Jg.: 83-190; Mainz.

— \& Müller, H. (1996): The Kärlich Interglacial site and its correlation with the Bilshausen sequence. In: Turner, Ch. (Ed.): The early Middle Pleistocene in Europe. - 187-193; Balkema, Rotterdam.

BoeNIGK, W. (1978): Gliederung der altquartären Ablagerungen in der Niederrheinischen Bucht. - Fortschr. Geol. Rheinld. u. Westf., 28: 135-212; Krefeld.

- (1995a): Terrassenstratigraphie des Mittelpleistozän am Niederrhein und Mittelrhein. - Mededelingen Rijks Geologische Dienst, 52: 71-81.

- (1995b): Central Upland margin Traverse. - In: SCHIRMER W. (ed.): Quaternary field trips in Central Europe. - Vol. 1, 559 - 598; München.

— \& Frechen, M. (1998a): Zur Geologie von Kärlich Hauptwand. - Röm. Germ. Zentralmuseum; im Druck: Mainz.

— \& Frechen, M. (1998b): Zur Geologie von KärlichSeeufer. - Mainzer Geowissenschaftl. Mitteilungen, im Druck; Mainz.

- Frechen, M. \& Schweitzer, U. (1998): Mikromorphologische Untersuchungen an Paläoböden aus Koblenz-Metternich und Kärlich, Rheinland.- Mainzer Geowissenschaftl. Mitteilungen, im Druck; Mainz.

- \& Heye, D., Schirmer, W. \& Brunnacker, K. (1974): Paläomagnetische Messungen an vielgliedrigen Quartär-Profilen (Kärlich/Mittelrhein und Bad Soden i. Taunus). - Mainzer Naturw. Arch., 12: 159 168; Mainz.

Bogatrd, C. v. D., BogaArd, P. v. D. \& SChmincke, H.-U. (1989): Quartärgeologisch-tephrostratigraphische Neuaufnahme und Interpretation des Pleistozänprofils Kärlich. - Eiszeitalter und Gegenwart, 39: 62-86; Hannover.

BogaARd, P. v. d. \& SCHMincke, H.-U. (1988): Aschelagen als quartäre Zeitmarken in Mitteleuropa.- Die Geowissenschaften, 6: 75-84; Weinheim.

— \& Schmincke, H.-U. (1990): Die Entwicklungsgeschichte des Mittelrheinraumes und die Eruptionsgeschichte des Osteifel-Vulkanfeldes. - In: ScHIRMER, W. (Hrsg.): Rheingeschichte zwischen Mosel und Maas. - deuqua-Führer, 1: 166-190; Hannover.

Bosinski, G., Brunnacker, K., Lanser, K.P., Stephan S., Urban, B. \& Würges, K. (1980): Altpaläolithische Funde von Kärlich, Kreis Mayen-Koblenz (Neuwieder Becken). - Arch. Korrespondenzbl., 10: 295314; Mainz.

Brunnacker, K. (1971): Beiträge zur Stratigraphie des Quartär-Profils von Kärlich am Mittelrhein. - Mainzer naturwiss. Archiv, 10: 77-100; Mainz.

Brunnacker, K. \& Boenigk W., Koči, A. \& Tillmanns, W. (1976): Die Matuyama/Brunhes-Grenze am Rhein und an der Donau. - N. JB. Geol. Paläont. Abh., 151: $358-378$; Stuttgart.

Brunnacker, K., Löhr, H., Boenigk, W., Puissegur, J. \& Poplin, F. (1975): Quartär-Aufschlüsse bei Ariendorf am unteren Mittelrhein. - Mainzer Naturwiss. Archiv, 14: 93-141; Mainz.

Brunnacker, K., Streit, R. \& Schirmer, W. (1969): Der Aufbau des Quartär-Profils von Kärlich/Neuwieder Becken (Mittelrhein). - Mainzer Naturw. Arch., 8: 102-133; Mainz.
Brunnacker, K., Würges, K. \& Urban, B. (1980): Kärlich (Terrassenschotter mit Lößdeckschichten). - In: Tagung der Deutschen Quartärvereinigung, Aachen 1980, Exkursion 1: Mittel- und Niederrhein: 79-86; Köln.

Debuyst, R., Dejehet, F., Callens, F. \& Frechen, M. (1998): ESR-dating of tooth enamel from the Kaerlich-Seeufer site in Germany. - 5th ESR Conference in Obninsk, Russia (May 1998); Proceedings.

Frechen, J. \& Boom, G.v.d. (1959): Die sedimentpetrographische Horizontierung der pleistozänen Terrassenschotter im Mittelrheingebiet. - Fortschr. Geol. Rheinld. u. Westf. 4: 89-125; Krefeld.

- \& Heide, H. (1969): Tephrostratigraphische Zusammenhänge zwischen der Vulkantätigkeit im Laacher-See-Gebiet und der Mineralführung der Terrassenschotter am unteren Mittelrhein.- Decheniana, 122: 35-74; Bonn.

- \& Rosauer, E. A. (1959): Aufbau und Gliederung des Würm-Profils von Kärlich im Neuwieder Becken. Fortschr. Geol. Rheinld. u. Westf., 4: 267-282; Krefeld.

— \& Villwock, R. (1959): Die ältesten Quartärsedimente in der Tongrube Kärlich im Neuwieder Becken.Fortschr. Geol. Rheinland u. Westfalen, 4: 267-282; Krefeld.

Fromm, K. (1987): Paläomagnetische Bestimmungen zur Korrelation altpleistozäner Terrassen des Mittelrheins. - Mainzer Geowissenschaftliche Mitteilung, 16: 7-29; Mainz.

GaudzInSKI, S. (1998): Kärlich-Seeufer. Untersuchungen zu einer altpaläolithischen Fundstelle im Neuwieder Becken (Rheinland/Deutschland). - Jahrb. d. Röm.-Germ. Zentralmuseums Mainz, (im Druck).

- Bittmann, F., Boenigk, W., Frechen, M. \& KolfschoTEN, Th. v. (1996): Palaeoecology and Archaeology of the Kärlich-Seeufer Open-Air Site (Middle Pleistocene) in Central Rhineland, Germany. - Quaternary Research, 46: 319-334; Washington.

Koči, A., Schirmer, W. \& Brunnacker, K. (1973): Paläomagnetische Daten aus dem mittleren Pleistozän des Rhein-Main-Raumes. - N. Jb. Geol. Paläontol. Mh, 1973 (9): 545-554; Stuttgart.

Kolfschoten, Th. v. (1990): The evolution of the Mammal fauna in the Netherlands and the Middle Rhine Area (Western Germany) during the Late Middle Pleistocene. - Mededelingen Rijks Geol. Dienst, 43: 3-69; Heerlen.

- \& Richter, J. (1998): The smaller mammals from the early Middle Pleistocene sequence at Kärlich (Germany). - Röm. Germ. Zentralmuseum, im Druck; Mainz.

- Roth, G. \& Turner, E. (1990): Mollusken und Säugetierfauna aus der Tongrube Kärlich.- In: SCHIRMER, W. (Hrsg.): Rheingeschichte zwischen Mosel und Maas. - deuqua-Führer, 1: 70-74; Hannover.

- \& Turner, E. (1996): Early Middle Pleistocene mammalian faunas from Kärlich and Miesenheim I and their biostratigraphical implications. - In: C. Turner (Hrsg.): The Early Middle Pleistocene in Europe. 227-253; Rotterdam.

Kröger, K., Bogaard, P. v. D., Bittmann, F. \& Turner, E. (1991):Der Fundplatz Kärlich-Seeufer. Neue Untersuchungen zum Altpaläolithikum im Rheinland. Jahrb. d. Röm.-Germ. Zentralmuseums Mainz, 35: 111-135; Mainz. 
Lippolt, H. J., Fuhrmann, U. \& HradeTzKy, H. (1986): ${ }^{40} \mathrm{Ar} / 39 \mathrm{Ar}$ age determinations on sanidines of the $\mathrm{Ei}-$ fel volcanic field (Federal Republic of Germany): Constraints on age and duration of a Middle Pleistocene cold period. - Chemical Geology (Isotope Geoscience Section), 59: 187-204; Amsterdam.

Mordzıol, C. (1930): Das Tertiär- und Diluvialprofil von Kärlich.- Z. deutsch. geol. Ges., 82: 599-600; Berlin.

Mückenhausen, E. (1959): Die stratigraphische Gliederung des Löß-Komplexes von Kärlich im Neuwieder Becken. - Fortschr. Geol. Rheinld. u. Westf., 4, 283-300; Krefeld.

RAZI RAD, M. (1976): Schwermineraluntersuchungen zur Quartär-Stratigraphie am Mittelrhein. - Sonderveröff. Geol. Inst. Univ. Köln, 28: 164 S.; Köln.

RohdenburG, H. \& Semmel, A. (1971): Bemerkungen zur Stratigraphie des Würm-Lösses im westlichen Mitteleuropa. - Notizbl. Hess. L.-Amt Bodenforsch., 99: 246 - 252; Wiesbaden.

RotH, G. (1993): Mollusken aus dem Mittelpleistozänen „Kärlicher Interglazial“ der Tongrube bei Kärlich (Neuwieder Becken/Mittelrhein). - Jahrb. Röm. Germ. Zentralmus., 40: 75-102; Mainz.

SCHIrmer, W. (1970): Das jüngere Pleistozän in der Tongrube Kärlich am Mittelrhein. - Mainzer Naturwiss. Arch., 9: 257-284; Mainz.

- (1990): Kärlich - Forschungsstand 1990.- In: SCHIRMER, W. (Hrsg.): Rheingeschichte zwischen Mosel und Maas. - deuqua-Führer, 1: 60-67; Hannover.

- (1995): Stop 37: Clay pit of Kärlich. - In: Schimer, W. (Hrsg.): Quaternary field trips in Central Europe. Volume 1: 525-526; Verlag Dr. Friedrich Pfeil; München.
SCHMincke, H.-U. (1994): Vulkanismus im Laacher See Gebiet. - Geol. Vereinigung-Exkursionsführer Nr. 1, 1-52; Kiel.

SGIBNEV, V. (1998): Zur Frage der genetischen Zugehörigkeit der lößähnlichen Sedimente in der Tongrube Kärlich (Neuwied/Mittelrhein), - Röm. Germ. Zentralmuseum, im Druck; Mainz.

Steensma, K. \& Kolfschoten, Th. v. (1998): The Middle Pleistocene (Saalian) smaller mammals (insectivora, rodentia) from Ariendorf, central Rhineland, Germany. - Jahrb. Röm. Germ. Zentralmus.; im Druck, Mainz

Tauxe, L., Herbert, T., Schackleton, N. J. \& KoK, Y. S. (1996): Astronomical calibration of the MatuyamaBrunhes boundary: Consequences for magnetic remanence acquisitation in marine carbonates and the Asian Loess sequences. - Earth and Planetary Science Letters, 140: 133-146; Amsterdam.

Turner, E. (1989): Middle and Late Pleistocene macrofaunas of the Neuwied Basin region (Rhineland-Palatinate) of West Germany. - Diss. Univ. Birmingham, 480 S.; Birmingham.

Urban, B. (1978): Die Stellung des „Kärlicher Interglazial“ am Mittelrhein.- In: Nagl, H. (Hrsg.): Beiträge zur Quartär- und Landschaftsforschung. - 649-654; Festschr. J. Fink, Wien.

- (1983): Biostratigraphic correlation of the "Kärlich interglacial", Northwestern Germany. - Boreas, 12: 83-90; Oslo.

Vollbrecht, J. (1998): Archäologie. - Röm. Germ. Zentralmuseum; im Druck; Mainz.

Manuskript eingegangen am 14. November 1997 